\title{
BEATRIX KISS-GEOSITS*
}

\section{UNTERSUCHUNG DER FAKTOREN, DIE HINSICHTLICH DES BURN-OUTS VON WESTUNGARISCHEN PÄDAGOGEN VON BEDEUTUNG SIND}

\author{
(Erhalten: 28. Juli 2009; angenommen: 21. Oktober 2009)
}

\begin{abstract}
Erziehern und Lehrern ist klar, dass sie bei ihrer Arbeit ständig mit vielfältigen emotionalen Reaktionen der Kinder / Erwachsenen konfrontiert werden und sich mit dem Ursprung und den Auswirkungen dieser Reaktionen befassen müssen. Sie müssen die Faktoren kennen, die den erzieherischen Prozess, ihre Arbeit, erleichtern beziehungsweise erschweren, und wissen, wie diese Schwierigkeiten bewusst zu überwinden sind, und zwar so, dass sie selbst dabei sowohl im Beruf als auch im Privatleben seelisch und emotional funktionsfähig bleiben. Ihr wichtigstes Arbeitsmittel bei der helfenden Arbeit ist eindeutig ihre eigene Persönlichkeit. Es ist wichtig, dass sie in der Lage sind, anderen zu helfen, ebenso wichtig ist jedoch, dass sie auch sich selbst instand halten können - denn das sind die zwei Seiten ein und desselben Prozesses. Das Gefühlsleben eines Menschen wirkt sich auch auf andere aus - und ist somit nicht nur Privatsache -, ebenso wird sein innerer Zustand von anderen Menschen, deren Umständen und Lebensführung beeinflusst. In diesem Beitrag werden die Faktoren untersucht, die hinsichtlich des Burnouts von Pädagogen von Bedeutung sind, wobei einzelne Elemente der Berufswahl, die Interessen, die Bedürfnisse, die Beziehungen des Einzelnen zu seiner Umgebung, die Attitüde der Eltern bei der Erziehung, das berufliche Vorbild in der Familie und die Zufriedenheit mit der Berufswahl im Mittelpunkt stehen. Im Rahmen der Frage nach dem Zusammenhang zwischen Arbeit und seelischer Gesundheit wird untersucht, inwieweit fachliches Ansehen sowie finanzielle und moralische Anerkennung hinsichtlich des Burn-outs eine Rolle spielen, welche Bedeutung den Beziehungen zu den Kollegen und zum Vorgesetzten hinsichtlich des Burn-outRisikos zukommt und wie körperliche gesundheitliche Symptome (Arbeitsausfälle, Krankschreibungen) und seelische Gesundheit zusammenhängen. Die Untersuchung differenziert anhand der Variablen des Geschlechts, der Dauer der Tätigkeit in dem jeweiligen Beruf sowie des Vorgesetzten- beziehungsweise Untergebenenstatus.
\end{abstract}

\footnotetext{
* Beatrix Kiss-Geosits, Westungarische Universität, Károlyi Gáspár tér 4., H-9400 Szombathely, Ungarn; geosits.beatrix@mnsk.nyme.hu.
} 
Schlüsselbegriffe: Pädagoge, Berufswahl, Interessen, Motivation, Attitüde der Eltern bei der Erziehung, Burn-out, Burn-out-Risiko, fachliches Ansehen, finanzielle Anerkennung, moralische Anerkennung

\begin{abstract}
A Study of Factors Influencing Burnout in Teachers in Western Hungary: It is obvious to professionals employed in teaching and education that over the course of their work they continuously need to face a multitude of emotional reactions from children and adults, and need to address the reasons and effects of such. Thus, it is important for them to be knowledgeable of the factors that render easier or more difficult the education process itself, their work, and the conscious ways of combating these difficulties; this, while staying psychologically and emotionally functional both in their work and in their private life. In their helping profession, their most important tool is obviously their own personality. It is important for them to be able to help others, but it is equally important to be able to take care of themselves, these being the two sides of the same process. A person's emotional life affects others - therefore it is not simply a private issue - whereas others, their circumstances, and their way of life affect the state of their inner self. This study set the goal of examining the factors influencing burnout in teachers. It therefore focuses on certain elements of choosing a profession, interests, needs, the relationship between the individual and the environment, parental upbringing attitudes, career patterns in the family, and satisfaction about career choice. Through the question of work and mental health, the author examines the degree to which professional, financial, and moral recognition influence burnout, the impact of the relationship with colleagues and the manager on the risk of burnout, and the connection between symptoms of physical health (days off work / sick leave) and mental health. The author further refines the issue studied along factors of sex, years spent in the profession, and manager / employee status.
\end{abstract}

Keywords: teacher, career choice, interests, motivation, parental upbringing attitude, burnout, risk of burnout, professional, financial and moral recognition

\title{
1. Einleitung
}

Viele Fachleute halten die Mentalhygiene für „den wichtigsten Bereich der Vorbeugung gegen Urbanisierungs- und Zivilisationsschäden“" (FRENKL 2003).

Erziehern und Lehrern ist klar, dass sie bei ihrer Arbeit ständig mit vielfältigen emotionalen Reaktionen der Kinder/Erwachsenen konfrontiert werden und sich mit dem Ursprung und den Auswirkungen dieser Reaktionen befassen müssen. Sie müssen die Faktoren kennen, die den erzieherischen Prozess und ihre Arbeit erleichtern beziehungsweise erschweren, und wissen, wie diese Schwierigkeiten bewusst zu überwinden sind, und zwar so, dass sie selbst dabei sowohl im Beruf als auch im Privatleben seelisch und emotional funktionsfähig bleiben. Das wichtigste Arbeitsmittel bei der helfenden Arbeit ist eindeutig ihre eigene Persönlichkeit. Es ist wichtig, dass sie in der Lage sind, anderen zu helfen, ebenso wichtig ist jedoch, dass sie auch sich selbst instand halten können - denn das sind die zwei Seiten ein und desselben Prozesses. Das Gefühlsleben eines Menschen wirkt sich auch auf andere aus - und ist somit nicht 
nur Privatsache -, ebenso wird sein innerer Zustand von anderen Menschen, deren Umständen und Lebensführung beeinflusst.

Es gibt relativ wenige umfassende Publikationen zu den Fragen der seelischen Gesundheit von Pädagogen (die Forschung konzentriert sich eher auf die Probleme der in der Pflege, der Krankenversorgung und im sozialen Bereich Tätigen), obwohl die Mentalhygiene der Pädagogen angesichts der gegenwärtigen Veränderungen in Wirtschaft und Gesellschaft sowie im Bildungswesen große Aktualität besitzt.

Die Kürzung der Mittel für Bildung und Unterricht und der normativen Zuschüsse je Schüler, die Minimierung der Zuschüsse für nichtstaatliche Einrichtungen, der Rückgang der Schülerzahlen, die größeren Klassen, die höhere Stundenzahl der Lehrer und die aufgrund dieser Umstände erfolgenden Schulschließungen haben Auswirkungen auf die Voraussetzungen und die Belastung der im Unterrichtswesen Tätigen. Es sind auch Probleme des persönlichen Rollenverlusts der Fachkräfte in den Einrichtungen für Erziehung und Unterricht zu beobachten. Eine wichtige Frage der nahen Zukunft wird sein, ob sich die Ausbildung der Lehrer im Bologna-System verlängert, da sie ihren Beruf nur mit einem Magister/Master in zwei Fächern ausüben dürfen.

Dieser Beitrag befasst sich mit der Lage der Pädagogen sowie den Faktoren, die ihre seelische Gesundheit gefährden. Es werden Antworten auf die Fragen gesucht, wie es um die seelische Gesundheit der Pädagogen steht und ob die verschiedenen Segmente der Berufswahl für ihre seelische Gesundheit von Bedeutung sind.

In welchem Umfang sind bei Erziehern und Lehrern Anzeichen des Burn-outs zu beobachten, und inwieweit stehen ihnen Möglichkeiten der seelischen Erholung und der Aufrechterhaltung der seelischen Gesundheit zur Verfügung?

\subsection{Die Segmente der erfolgreichen Berufswahl}

\subsubsection{Die Bedürfnisse}

Laut Ann Roe sind die wichtigsten Motivationsfaktoren für die Berufswahl die Bedürfnisstruktur und die auf dieser basierenden Interessen, durch welche die Berufsorientierung junger Leute unmittelbar beeinflusst wird. Die Bedürfnisse entwickeln sich kontinuierlich weiter, und zwar in Abhängigkeit von der Atmosphäre in der Familie (SZILÁGYI 1993). Die Grundlage der Entwicklung der Bedürfnisse ist die emotionale Position des Kindes in der Familie, die in der frühen Kindheit wurzelt (HoRVÁTH-SZABÓ 2007). In dieser Hinsicht ist auch die Atmosphäre der Eltern-Kind-Beziehung von Bedeutung.

Die Berufswahl lässt sich direkt oder indirekt überwiegend mit der Befriedigung der Bedürfnisse beziehungsweise anhand der Reduktion der Bedürfnisenergien erklären. Eine ähnlich bedeutende Rolle wie die Bedürfnisse nehmen bei der Gestaltung der fachlichen und zwischenmenschlichen Beziehungen die Triebe beziehungsweise die von den Trieben gesteuerten Energien ein (SZILÁGYI 2000). 


\subsubsection{Beziehung zwischen dem Individuum und seiner Umgebung}

Die Berufswahl wird durch die Beziehung zwischen dem Individuum und seiner Umgebung und durch die unterschiedliche Akzeptanz in dieser Relation bestimmt. Diesbezüglich weisen die fachlichen Meinungen Gewichtungsunterschiede auf, die durch wirtschaftliche und Umweltfaktoren, die Persönlichkeit, die Entscheidungsdominanz, durch Fähigkeiten, Neigungen, Werte und Traditionen nuanciert werden (SZILÁGYI 1997).

Laut Roe können sechs elterliche Verhaltensweisen die Berufswahl beeinflussen:

- die übermäßig beschützende, das Sammeln von Erfahrungen verhindernde, niedrige Anforderungen stellende und verwöhnende;

- die überfordernde, Bedürfnisse befriedigende und leistungsorientierte,

- die an der Entwicklung des Kindes ausgerichtete, akzeptierende, Bedürfnisse angemessen befriedigende und zur Selbstständigkeit erziehende;

- die gelegentlich, nach dem Zufallsprinzip und stimmungsabhängig akzeptierende;

- die emotional zurückweisende, nur die physiologischen Bedürfnisse befriedigende, die keinen Schutz, keine Liebe und keine Anerkennung bietet;

- die vernachlässigende, die physiologischen Bedürfnisse nicht befriedigende.

Roe zufolge führen die ersten drei Verhaltensweisen zu einer persönlichen Orientierung (beispielsweise helfende Berufe) und die folgenden drei zu einer sachlichen Orientierung des Kindes (zum Beispiel naturwissenschaftliche Berufe) (SZILÁGYI 1993; GEOSITS \& KARDOS 1999).

\subsubsection{Motivation}

Für eine humanspezifische Handlung, wie es auch die Berufswahl ist, kann es zahlreiche Auslöser, Motivationen geben. Bei der Untersuchung der Motivation stehen der Hintergrund und die Beweggründe menschlicher Handlungen im Mittelpunkt. Es geht darum, was für den Beginn und die Steuerung der Handlung sowie ihre Fortsetzung bis zu dem Punkt, an dem die zielgerichtete Handlung zur Befriedigung der Motivation (Freude, Gefühl der Befriedigung, Gleichgültigkeit oder Sättigungsgefühl) des Individuums führt, verantwortlich ist (DANCS 1989; N. KOLLÁR \& SZABÓ 2004).

Die Motivationen zielen - je nachdem, ob sie extrinsisch oder intrinsisch sind auf einen Zielgewinn ab, oder das Ziel ist die Freude an der Handlung als solcher. Intrinsische Motivationen können sein:

- Kompetenzmotivation (effektiver Umgang mit dem Umfeld),

- Aneignungsmotivation (Weiterentwicklung von Fertigkeiten und Fähigkeiten),

- Interesse (situationsspezifisches oder dauerhaftes persönliches Interesse) (N. KOLLÁR \& SZABÓ 2004). 


\subsubsection{Interesse}

Eine der zentralen Fragen bei der Berufswahl ist das Interesse, da dieses Motiv das Individuum dazu veranlasst, „zum Zwecke des Erwerbs von Kenntnissen in Bezug auf eine Sache oder ein Phänomen eine anhaltende und ausdauernde Aktivität“" auszuführen beziehungsweise einen Bereich der ihn umgebenden Wirklichkeit intensiver zu studieren (KLEIN 1990).

Ein besonderer Bereich innerhalb des Interesses ist das Arbeitsinteresse, das die Hinwendung des Individuums zur Arbeitsveranlagung sowie seine dauerhafte, prozesshafte Beziehung zur Arbeit bestimmt. Larcebeau hat in seinen Untersuchungen gezeigt, dass sich die Kategorien des primären Interesses bis zum 10. Lebensjahr herausbilden, während des gesamten Lebens eines Menschen bestehen bleiben und hinsichtlich der Aufrechterhaltung der seelischen Gesundheit eine ausgleichende Rolle spielen (CSIRSZKA 1985). Die Ermittlung des Arbeitsinteresses, von dem es fünf Grundtypen (direktiv, innovativ, sachlich, systematisch und sozial) gibt, ermöglicht die Einordnung der Beziehung des Individuums zur Arbeit (SZILÁGYI 2000).

\subsubsection{Zufriedenheit mit der Berufswahl}

Die Zufriedenheit mit der Berufswahl hängt vor allem davon ab, inwieweit das Individuum einen geeigneten Anwendungsbereich für seine Fähigkeiten, sein Interesse und sein Wertsystem findet. Die Zufriedenheit mit dem jeweiligen Beruf wird selbstverständlich über eine bestimmte Form der Arbeit erreicht, die auf der allgemeinen Ebene durch den Lebensstil und die konkrete Arbeitssituation bestimmt wird (BAGDY 1997).

Die Identifizierung mit dem Beruf ist ein Relationsbegriff, der die Qualität der Kongruenz der Anforderungen des Berufs mit dem die Arbeit ausführenden Menschen und seinen dominanten Persönlichkeitsmerkmalen ausdrückt. Dieser Relationsbegriff vermittelt eine dynamische Widerspiegelung des Prozesses der Laufbahn und der Persönlichkeit; die Identifizierung mit dem Beruf ist also ein dynamischer Prozess. Empathie und professionalisierter Altruismus sind Indikatoren für eine reife Identifizierung mit dem Beruf (RITOÓKNÉ 2000).

\subsection{Die Bedeutung der Arbeit für die seelische Gesundheit}

Ein wichtiges Kriterium der Gesundheit des Menschen ist die Arbeitsfähigkeit, die seelische Gesundheit kann jedoch auch durch die Zufriedenheit mit der Arbeit gestärkt werden (BODNÁR 1998). Die Zufriedenheit hängt von äußeren und inneren Faktoren ab: der Art des Berufs, dem Abwechslungsreichtum des Inhalts der Arbeit, der Minimierung der Wiederholung von Arbeitsabläufen, der Position in der Hierarchie, der finanziellen Anerkennung sowie der fachlichen und der moralischen Anerkennung.

Zur Wahl eines helfenden Berufes kann das Individuum durch verschiedene äußere und innere Kräfte motiviert werden. Diese erkennt das Individuum zu Beginn 
der Ausübung des Berufs nicht immer, sie werden ihm zu diesem Zeitpunkt nicht in jedem Fall bewusst. Eine spätere Bewusstwerdung kann ein Trauma auslösen, das Ausbleiben der Bewusstwerdung kann dazu führen, dass der Helfer schnell abstumpft und gleichgültig wird. Ein guter Helfer kann das mit sich ausmachen, und wenn es ihm gelingt, an diesem Problem zu arbeiten, kann er die Gefahr eines Burn-outs vermeiden.

\subsection{Burn-out}

Burn-out-Syndrom ist ein Zustand der physischen, emotionalen und mentalen Erschöpfung, der infolge von chronischer emotionaler Belastung und chronischem Stress eintritt, mit dem Gefühl der Hoffnungslosigkeit und Inkompetenz sowie dem Verlust von Zielen und Idealen einhergeht und durch negative Attitüden bezüglich der eigenen Person, der Arbeit und anderer Menschen gekennzeichnet ist (FREUDENBERGER 1974).

Nach der Definition von Freudenberger und Richelson ist Burn-out „ein Zustand der Erschöpfung und der Frustration, der durch das Festhalten an einer Lebensform oder einer Bezogenheit ausgelöst wird, die nicht die erwartete Belohnung gebracht hat" (FREUDENBERGER 1974, 161). EDELWICH \& BRODSKY (1980) hingegen definieren das Burn-out als progressiven Verlust des Idealismus, der Energie, des Zielbewusstseins und des Sorgens für andere.

\subsubsection{Gründe für ein Burn-out}

In den letzten Jahrzehnten war es Gegenstand zahlreicher Forschungen, dass hinsichtlich der körperlichen und seelischen Gesundheit neben den chemischen, biologischen und physikalischen Faktoren auch die psychosozialen Stressfaktoren der Arbeit von Bedeutung sind. Der Ausgangspunkt der Untersuchungen war die Stresstheorie nach Selye (SALAVECZ 2008).

Burn-out ist das Resultat dauerhafter oder wiederholter emotionaler Belastung im Zusammenhang mit langfristigen intensiven Anstrengungen für andere Menschen. Der Begriff des Burn-outs wird nur im Zusammenhang mit helfenden Berufen verwendet, da Menschen mit solchen Berufen, wenn sie ihre helfende Identität ernst nehmen, ein erhöhtes Burn-out-Risiko haben. Infolge eines hohen Maßes an Stress, Situationen, in denen verantwortungsvolle Entscheidungen getroffen werden müssen, und großer emotionaler Belastung kann es zu einer schweren emotionalen Erschöpfung kommen (GALlus 1996).

Ditsa Kafry hat im Jahr 1990 in einer Untersuchung mit 4.000 Teilnehmern drei wichtige Ursachen des Burn-outs ermittelt:

- emotionale Überlastung;

- bestimmte Persönlichkeitsmerkmale, die auch bei der Berufswahl entscheidend waren;

- eine klientenzentrierte Orientierung. 
Die Erschöpfung zeigte sich bei den Betroffenen in Form von körperlichen, geistigen und emotionalen Symptomen. Zu den körperlichen gehörten chronische Müdigkeit, Schwäche und Lustlosigkeit, Krankheitsanfälligkeit sowie Veränderungen der Essgewohnheiten und des Körpergewichts. Im emotionalen Bereich traten vor allem Erschöpfung, Niedergeschlagenheit, Hilflosigkeit, Hoffnungslosigkeit und Aussichtslosigkeit auf. Der Zustand der geistigen Erschöpfung ist durch eine negative Einstellung der Betroffenen gegenüber der eigenen Person, der Arbeit und dem Leben im Allgemeinen gekennzeichnet. Auf der Beziehungsebene ist gleichgültiges und unpersönliches Verhalten gegenüber denjenigen, denen geholfen werden soll, charakteristisch (ÓNODY 2001).

A.R. Barth nennt auf die Frage nach den Gründen des Burn-outs ebenfalls drei ursächliche Faktoren:

- ein hohes Maß an Stress und eine große Belastung, die zu emotionaler Erschöpfung führen;

- geringe Motivation bei der Arbeit und ein geringes Maß an Zufriedenheit am Arbeitsplatz, die zum Leistungsabfall führen;

- eine Verschlechterung der Beziehungen am Arbeitsplatz, die eine Dehumanisierung zur Folge hat.

Burn-out ist die Erschöpfung der empathischen Kapazität. Der Helfer kann seinen Beruf nicht effizient ausüben, wenn er den Klienten als Gegenstand behandelt. Ein Burn-out ist keine notwendige Begleiterscheinung des Altruismus, sondern eine mögliche Folge desselben, die nur eintritt, wenn die empathische Kapazität erschöpft wird (ÓNODY 2001).

\subsubsection{Symptome des Burn-outs}

Die Symptome des Burn-outs treten in drei Bereichen auf:

- emotionale Erschöpfung: Müdigkeit, chronische Erschöpfung, Schlaflosigkeit, Krankheitsanfälligkeit, diffuse körperliche Beschwerden;

- Depersonalisierung und Dehumanisierung: negatives, zynisches Verhältnis zu den Kollegen, negative Gefühle gegenüber Patienten, Klienten, Kunden, Schuldgefühle, ein Sichzurückziehen, Abwehrhaltung, Reduzierung der Arbeit;

- Abfall der persönlichen Leistung: Erleben von Erfolglosigkeit und Ohnmacht, fehlende Anerkennung, Gefühl der Unzulänglichkeit und der Überlastung (PÁLFFY 2003).

Gábor HÉzSER hat die Symptome des Burn-outs vom C. Cherniss in seinem Buch folgendermaßen systematisiert:

Um mit der täglichen Arbeit zu beginnen, muss ein großer innerer Widerstand überwunden werden. Die Betroffenen beherrscht ein Gefühl der Erfolglosigkeit, der Gereiztheit, der Gleichgültigkeit und des Sichzurückziehens, sie klagen über übermäßige Müdigkeit und Erschöpfung während und nach der Arbeit. Sie müssen immer größere Anstrengungen unternehmen, um geduldig und mit Empathie mit den Schülern umzugehen, die Hilfe 
brauchen; manche Schüler gehen dem Helfenden regelrecht auf die Nerven. Sie verrichten ihre Arbeit mechanisch, zeigen übermäßiges Verständnis für Klienten, die Beruhigungsmittel nehmen, und meiden Fallbesprechungen mit den Kollegen. Sie klagen regelmäßig über Schlafstörungen, Appetitlosigkeit, haben eine banale Erkrankung nach der anderen, häufig Kopfschmerzen, Verdauungs- und Magenbeschwerden. Sie reagieren auch auf positive Veränderungen am Arbeitsplatz negativ, es ist ein sprunghafter Anstieg der Gefahr von Alkoholismus und Medikamentenabhängigkeit zu beobachten, familiäre und Eheprobleme werden zum Dauerzustand. Freundschaften und soziale Kontakte werden zunehmend vernachlässigt, die Betroffenen ziehen sich zurück. (HÉZSER 2001, 81, eigene übers.) ${ }^{1}$

\subsubsection{Die Berufsgruppen mit dem größten Burn-out-Risiko}

$\mathrm{Zu}$ einem Burn-out kann es bei praktisch allen Berufsgruppen kommen, bei denen die interpersonellen Prozesse bipolar sind. Die Betreffenden werden mit der Lösung oder Unlösbarkeit von Problemen konfrontiert, bei denen die bewusste und aktive Kooperation einer anderen Person erforderlich ist. Wenn diese Kooperation nicht zustande kommt, etwa durch aktiven oder passiven Widerstand oder aus einem objektiven Grund (zum Beispiel Alter, Zurückgebliebenheit, psychiatrische Erkrankung, schwere physiologische Krankheitsverläufe, Lebensrettung), verursacht dies Stress.

Burn-out-gefährdet sind vor allem die Angehörigen der ,heiligen Berufe“: Psychologen, Pfarrer, Ärzte, Rechtsanwälte, Pädagogen und die Angehörigen der helfenden Berufe im Allgemeinen.

Gefährdet sind auch Führungskräfte, Geschäftsleute und Politiker.

Früher behaupteten die Forscher, es gebe Menschen, die nicht zur primären Risikogruppe gehören, und zwar diejenigen, die eine friedliche, ruhige Tätigkeit ausüben wie Schäfer, Fischer, Handwerker, Künstler und all diejenigen, die sich über kürzere oder längere Zeit vom aktiven oder passiven Widerstand anderer Menschen oder den objektiven Ursachen, die Widerstand auslösen, isolieren können. Die Forschungen am Ende des 20. Jahrhunderts haben jedoch gezeigt, dass es unabhängig von seinen Arbeitsaufgaben bei fast jedem Menschen zu einem Burn-out kommen kann (COLEMAN et al. 2006).

\footnotetext{
1 'A mindennapi munka megkezdése nagy belső ellenállás leküzdését igényli. Eluralkodik a sikertelenség, ingerültség, közömbösség és a visszahúzódás érzése, a munkavégzés alatt és a munka után túlzott fáradtságról, kimerültségröl panaszkodnak. Egyre nagyobb erőfeszítésekbe kerül, hogy türelemmel empatikusan bánjanak a segítségre szoruló tanulókkal, vannak akik kifejezetten idegesítik a segítő foglalkozást végzőt. Munkájukat sematikusan végzik; túlzott megértést tanúsítanak a nyugtató szereket szedő kliensekkel szemben, kerülik a kollegiális esetmegbeszéléseket. Rendszeresen panaszkodnak alvászavarokról, étvágytalanságról, sorozatossá válnak a banális betegségek, gyakori a fejfájás, az emésztési és gyomorpanaszok. Pozitív munkahelyi változásokra is negatívan reagálnak, ugrásszerūen megnő az alkoholizmus, a gyógyszerfüggőség veszélye, állandósulnak a családi és házassági problémák. Baráti és szociális kapcsolataikat egyre jobban elhanyagolják visszahúzódnak.'
} 


\subsection{Die Bedeutung der seelischen Gesundheit bei der erzieherischen und lehrenden Tätigkeit}

Barth zufolge zeigen etwa $25 \%$ der Lehrer, die seit 5 Jahren im Dienst sind, Anzeichen eines Burn-outs (ÓNODY 2001).

Die seelische Gesundheit von Erziehern und Lehrern ist also besonders gefährdet. Sie haben ständig mit Menschen zu tun, sie müssen sich bei ihrer Arbeit ununterbrochen konzentrieren und werden emotional beansprucht. Da sie wenig Rückmeldungen erhalten und es wenig greifbare Anhaltspunkte für definitive Ergebnisse gibt, haben sie sehr wenige Erfolgserlebnisse. Bei ihrer Arbeit, die praktisch nie ein Ende hat, werden die verschiedensten, oftmals gegensätzlichen Erwartungen an sie gestellt.

\section{Beschreibung des Forschungsprojekts}

\subsection{Methode}

Es wurde ein Fragebogenpaket zum selbstständigen Ausfüllen erarbeitet, das von Januar bis April 2008 auf dem Server der Westungarischen Universität bereitgestellt wurde.

Im ersten Teil ging es um den Lebensstil, sportliche Aktivitäten und die berufliche Motivation. Die Untereinheiten dieses ersten Teils waren: Informationen zum Beruf, Motivationen für die Berufswahl (Interesse, Attitüde der Eltern, familiäres Vorbild), Fragen zum sozioökonomischen Status und zur täglichen Arbeit (Atmosphäre am Arbeitsplatz, Anerkennung, Freizeit, Gesundheitszustand, Möglichkeiten der Hilfestellung) sowie zum Arbeitsinteresse.

Im zweiten Teil sollten die Symptome des Burn-outs unter Verwendung des von Hézser erarbeiteten Fragebogens zur persönlichen Belastung ermittelt werden, dem Maslachs Burnout Inventory zugrunde liegt.

Die Forschungsdaten wurden mit dem Tabellenkalkulationsprogramm Excel for Windows und der Statistiksoftware SPSS 17.0 for Windows verarbeitet. Die Antworten auf die einzelnen Fragen des Fragebogens sind überwiegend kategorisierte Variablen. Die Ergebnisse werden als eindimensionale Häufigkeitsverteilungen und in Kontingenztabellen dargestellt, die Zusammenhänge zwischen den Variablen wurden mit dem Chi-Quadrat-Test analysiert.

\subsection{Stichprobe}

Der mentalhygienische Zustand von Pädagogen wurde im Jahr 2004 von einer Forschungsgruppe des ungarischen Landesinstituts für öffentlichen Unterricht an einer repräsentativen landesweiten Stichprobe untersucht. Die günstigen landesweiten Ergebnisse sollten im gegenständlichen Forschungsprojekt bestätigt werden. An dem Projekt haben sich die Unterrichts- und Erziehungseinrichtungen der ungarischen 
Region Westtransdanubien beteiligt. Von den 568 übermittelten Fragebögen wurden 363 zurückgesandt. Der Anteil der zurückgesandten Fragebögen betrug 64\%. 25\% der zurückgesandten Fragebögen wurden von Männern ausgefüllt. 33\% der Befragten waren im Bereich der Erziehung und 67\% als Lehrkräfte tätig (Abbildung 1).

\section{Ergebnisse}

Bei der Verarbeitung der zurückgesandten Fragebögen fiel auf, dass die Dauer der Ausübung des Berufs bei den Männern vollkommen mit der Dauer der Beschäftigung am aktuellen Arbeitsplatz übereinstimmte, während bei den Frauen ein beträchtlicher Arbeitsplatzwechsel zu verzeichnen war (48\% der Pädagoginnen übten ihren Beruf seit mehr als 21 Jahren aus, an ihrem aktuellen Arbeitsplatz arbeiteten nur 27\% seit dem Beginn der Ausübung des Berufs). Männer sind stärker burn-out-gefährdet als ihre Kolleginnen (Sig. 0,178). 24\% der Befragten hatten eine leitende Position inne, und zwar 59\% der Männer und 13\% der Frauen (Abbildung 2).

Der Anteil der Führungskräfte stieg in der untersuchten Stichprobe proportional zum Alter (Abbildung 3). Es bestand ein signifikanter Zusammenhang zwischen dem Burn-out-Risiko und dem Untergebenenstatus (Sig. 0,011). Von den Befragten in einer leitenden Position waren 13,3\%, von denen mit Untergebenenstatus 27,6 \% burn-outgefährdet.

Die Antworten auf die Fragen nach der Attitüde der Eltern ergaben Folgendes: $6 \%$ der Befragten berichteten über eine übermäßig beschützende Attitüde der Eltern. $18 \%$ bewerteten ihre Eltern als überfordernd, $64 \%$ als akzeptierend. $9 \%$ gaben an, ihre Eltern seien gelegentlich akzeptierend, $1 \%$ bewerteten die Attitüde ihrer Eltern als emotional zurückweisend und nur die physiologischen Bedürfnisse befriedigend, und $5 \%$ hatten vernachlässigende Eltern. Bei der erzieherischen Attitüde der Eltern zeigten sich Geschlechterunterschiede (Abbildung 4).

Von den beiden Elternteilen hatte die Mutter den größten Einfluss auf die Berufswahl. Bedeutend war außerdem der Einfluss von Tanten und Schwestern. Als Antwort auf eine offene Frage gaben 53\% der Befragten an, sich als Kinder einen pädagogischen Beruf (Kindergärtnerin, Grundschullehrer/in, Lehrer/in, Sportlehrer/in, Tanzlehrer/in, Trainer/in, Pädagoge usw.) gewünscht zu haben. 35\% der Befragten hatten ein familiäres Vorbild. Bei $20 \%$ der Pädagogen stimmte der Beruf sowohl mit dem familiären Vorbild als auch mit dem Berufswunsch in der Kindheit überein. Der Berufswunsch Pädagoge und das familiäre Vorbild waren bei den Männern häufiger als bei der Gesamtheit der Befragten (Abbildung 5).

Hinsichtlich der Richtung des Interesses der Pädagogen in der Kindheit dominierten „direktiv“ (29\%) und „sozial“ (26\%). Bei den untergebenen Pädagogen überwog das soziale Interesse (Abbildung 6 ). Es bestand ein signifikanter Unterschied (Sig. 0,027) zwischen der Interessenausrichtung in der Kindheit von Pädagogen in einer leitenden Position und solchen mit Untergebenenstatus. Die Richtung „direktiv“ zeigte eine deutliche Dominanz bei den Pädagogen in einer leitenden Position (40\%), das soziale und das innovative Interesse waren (mit 23\% und 20\%) fast gleich stark 


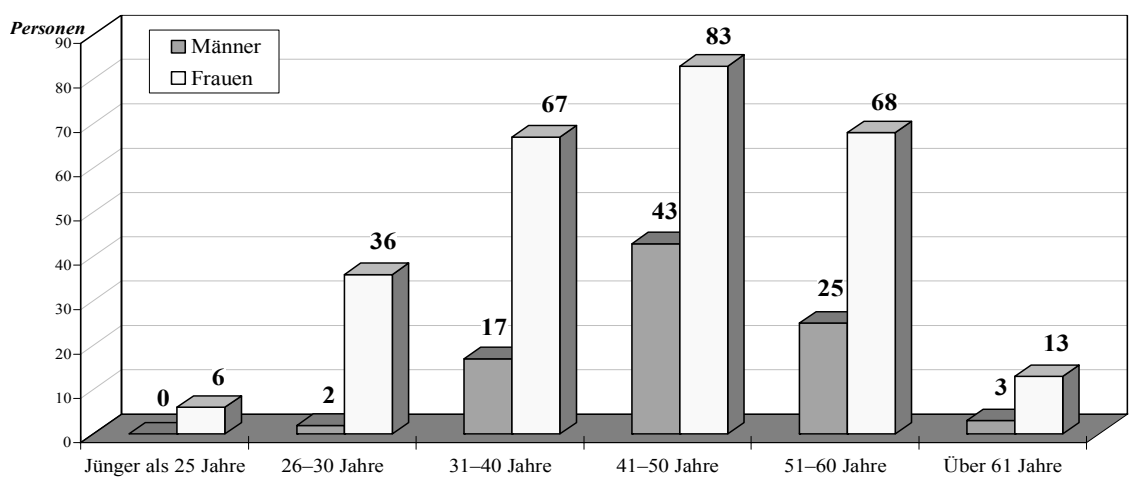

Abbildung 1

Anteil von Männern und Frauen nach Altersgruppen

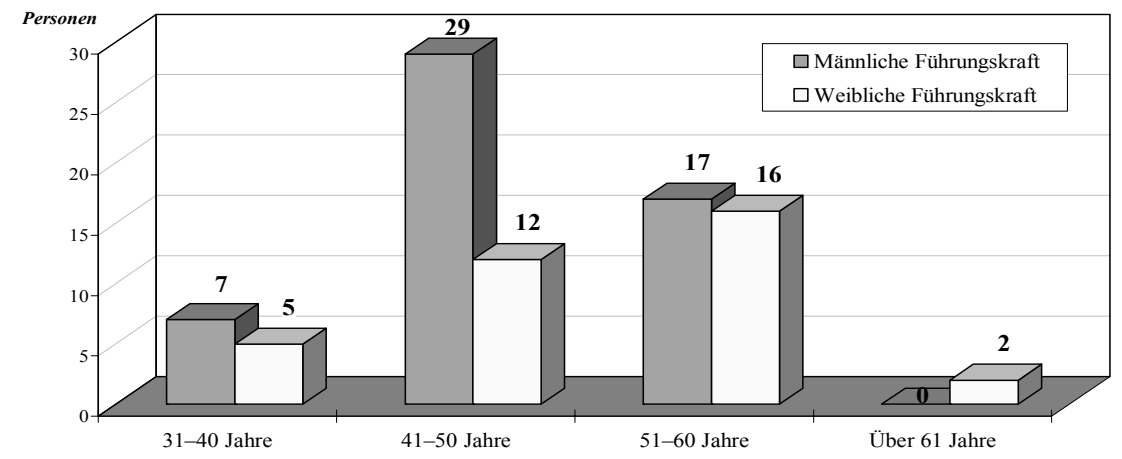

Abbildung 2

Altersgruppenverteilung männlicher und weiblicher Führungskräfte

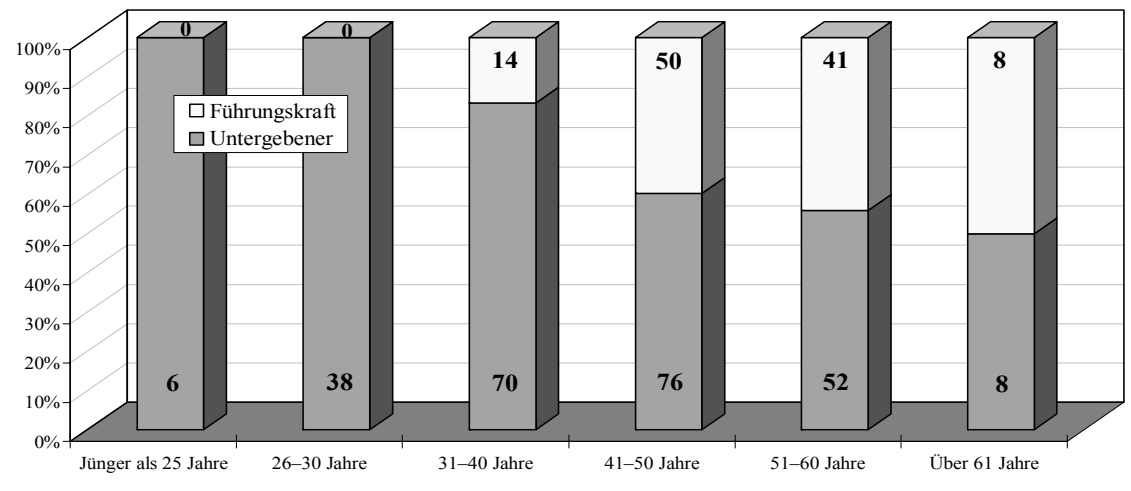

Abbildung 3

Verteilung der Untergebenen und der Führungskräfte nach Altersgruppen 


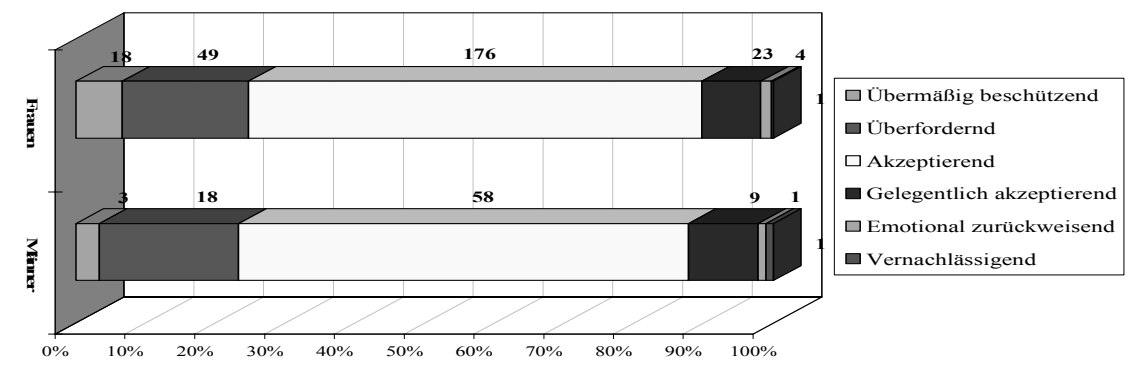

Abbildung 4

Die erzieherische Attitüde der Eltern nach Geschlechtern

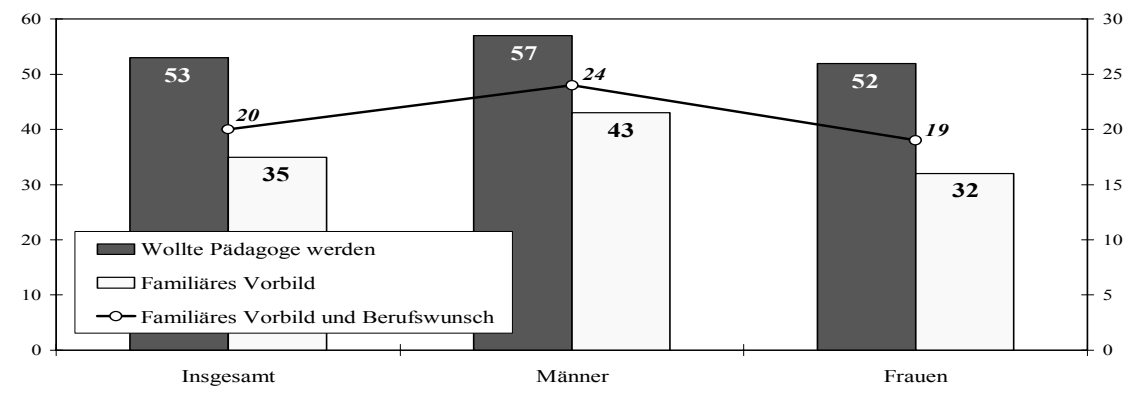

Abbildung 5

Häufigkeit des Berufswunsches in der Kindheit und des familiären Vorbildes

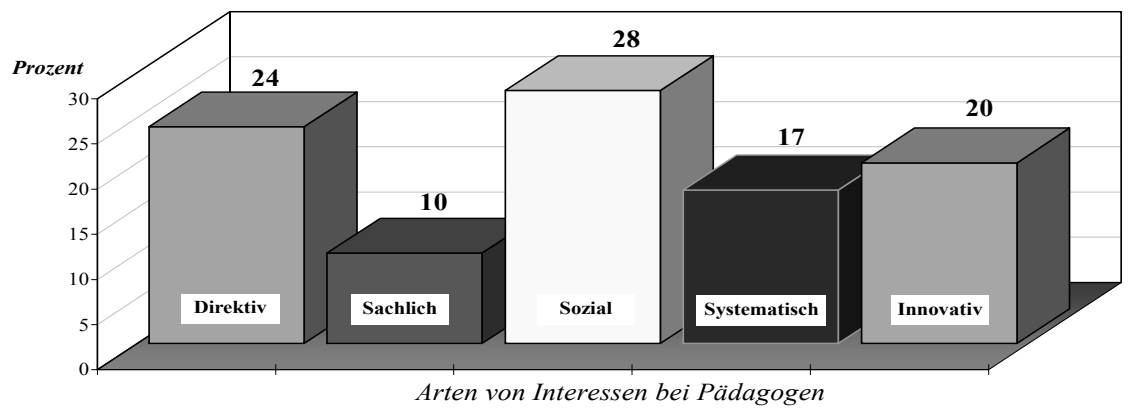

Abbildung 6

Interessen in der Kindheit bei Pädagogen mit Untergebenenstatus

vertreten, während das sachliche und das systematische Interesse eine geringe Häufigkeit zeigten (jeweils 8\%). Bei den Interessen wurden auch Unterschiede zwischen den Geschlechtern festgestellt. Bei den Männern überwogen die Richtungen „direktiv“ und „innovativ“, bei den Frauen stand das soziale Interesse an erster Stelle, gefolgt von der Richtung ,direktiv“. 
Die Untersuchung des Zusammenhangs zwischen der Dauer der Ausübung des Berufs und dem Burn-out ergab überraschenderweise, dass sich die Burn-out-Gefährdung mit der zunehmenden Dauer der Ausübung des Berufs nicht nur nicht erhöht, sondern sogar verringert (Tabelle 1).

Tabelle 1

Kontingenztabelle zur Dauer der Ausübung des jeweiligen Berufs und zur Burn-out-Gefährdung

\begin{tabular}{|c|c|c|c|c|c|}
\hline & & \multicolumn{3}{|c|}{ Burn-out-Kategorien } & \multirow[b]{2}{*}{ Insgesamt } \\
\hline & & $\begin{array}{l}\text { Nicht } \\
\text { gefährdet }\end{array}$ & $\begin{array}{l}\text { Durch- } \\
\text { schnittlich }\end{array}$ & $\begin{array}{l}\text { Burn-out- } \\
\text { gefährdet }\end{array}$ & \\
\hline \multirow[t]{4}{*}{ 0-10 Jahre } & & 20 & 42 & 24 & 86 \\
\hline & $\begin{array}{l}\text { Dauer der Ausübung } \\
\text { des Berufs }\end{array}$ & $23,3 \%$ & $48,8 \%$ & $27,9 \%$ & $100,0 \%$ \\
\hline & Burn-out & $15,9 \%$ & $27,5 \%$ & $28,6 \%$ & $23,7 \%$ \\
\hline & Insgesamt & $5,5 \%$ & $11,6 \%$ & $6,6 \%$ & $23,7 \%$ \\
\hline \multirow[t]{4}{*}{ 11-20 Jahre } & & 34 & 28 & 23 & 85 \\
\hline & $\begin{array}{l}\text { Dauer der Ausübung } \\
\text { des Berufs }\end{array}$ & $40,0 \%$ & $32,9 \%$ & $27,1 \%$ & $100,0 \%$ \\
\hline & Burn-out & $27,0 \%$ & $18,3 \%$ & $27,4 \%$ & $23,4 \%$ \\
\hline & Insgesamt & $9,4 \%$ & $7,7 \%$ & $6,3 \%$ & $23,4 \%$ \\
\hline \multirow{5}{*}{$\begin{array}{l}\text { Mehr als } \\
21 \text { Jahre }\end{array}$} & & & & & \\
\hline & & 72 & 83 & 37 & 192 \\
\hline & $\begin{array}{l}\text { Dauer der Ausübung } \\
\text { des Berufs }\end{array}$ & $37,4 \%$ & $43,2 \%$ & $19,3 \%$ & $100,0 \%$ \\
\hline & Burn-out & $57,1 \%$ & $54,2 \%$ & $44,0 \%$ & $52,9 \%$ \\
\hline & Insgesamt & $19,8 \%$ & $22,9 \%$ & $10,2 \%$ & $52,9 \%$ \\
\hline \multirow[t]{4}{*}{ Insgesamt } & & 126 & 153 & 84 & 363 \\
\hline & $\begin{array}{l}\text { Dauer der Ausübung } \\
\text { des Berufs }\end{array}$ & $34,7 \%$ & $42,1 \%$ & $23,1 \%$ & $100,0 \%$ \\
\hline & Burn-out & $100,0 \%$ & $100,0 \%$ & $100,0 \%$ & $100,0 \%$ \\
\hline & Insgesamt & $34,7 \%$ & $42,1 \%$ & $23,1 \%$ & $100,0 \%$ \\
\hline
\end{tabular}

Nur ein Drittel der Befragten erhielten überhaupt keine Hilfe, und jeweils die Hälfte von ihnen gab an, sich fachliche Hilfe zu wünschen beziehungsweise diese nicht zu benötigen. Es ist interessant, dass ein signifikant größerer Teil der Erzieher und Lehrer in leitenden Positionen, 66\% (Sig. 0,00), an Fallbesprechungen teilnahm. Knapp 90\% der Führungskräfte hatten bereits an Selbsterfahrungsgruppen und Gruppen zur Persönlichkeitsentwicklung teilgenommen (Sig. 0,00).

Der Anteil derer hingegen, die die Supervision in Anspruch nehmen, ist bei Führungskräften und Untergebenen gleich. Von den Pädagogen, die fanden, sie würden fachlich anerkannt, waren nur $18 \%$ burn-out-gefährdet. Von den Pädagogen, die über fehlende fachliche Anerkennung berichteten, waren nur 18\% nicht burn-out-gefährdet 
und 43\% burn-out-gefährdet. Zusätzlich zur fachlichen Anerkennung verringerte auch die finanzielle Anerkennung das Burn-out-Risiko (15\%), wobei die fehlende finanzielle Anerkennung die Burn-out-Gefährdung nicht erhöhte (Sig. 0,005). Moralische und menschliche Anerkennung führte nicht zu einem niedrigeren Burn-out-Risiko, das Fehlen moralischer und menschlicher Anerkennung hingegen erhöhte die Burn-outGefährdung in beträchtlichem Maße (53\%, Sig. 0,00).

Bei den Befragten, die ihre Beziehung zu ihren Kollegen und Mitarbeitern als „harmonisch und durch gute Kooperation gekennzeichnet" bewerteten, und bei denen, die die Antwort „Die Kooperation ist im Allgemeinen gut" wählten, war die Burnout-Gefährdung gering (15\% und 27\%). Bei denjenigen, die die Antwort „Es gibt kleinere oder größere Auseinandersetzungen" wählten, betrug das Burn-out-Risiko bereits mehr als $40 \%$. Diejenigen, die ihre Beziehungen am Arbeitsplatz als ,konfliktreich, mit häufigen Auseinandersetzungen" bezeichneten, gehörten alle zur Gruppe der Burn-out-Gefährdeten. Die Qualität der Beziehung mit den Kollegen wirkt sich signifikant (Sig. 0,001) auf das Burn-out-Risiko aus.

Noch markanter wirkt sich die Qualität der Beziehung zu Führungskräften und Vorgesetzten auf die Burn-out-Gefährdung beziehungsweise das Fehlen derselben aus. Bei einer „harmonischen und durch gute Kooperation gekennzeichneten“ Beziehung zwischen Vorgesetztem und Untergebenem beträgt das Burn-out-Risiko von Pädagogen 10\%. Bei denen, die die Antwort „Die Arbeitsbeziehung ist im Allgemeinen gut“ wählten, betrug die Gefährdung schon 27\%. Bei der Antwort „Es gibt kleinere oder größere Auseinandersetzungen“ erhöht sich das Burn-out-Risiko auf über 30\% und bei „konfliktreich, mit häufigen Auseinandersetzungen“ auf über 40\%. Bei Angestellten, die angaben, Probleme erst gar nicht anzusprechen, da sie ohnehin keine Hilfe erhalten würden, betrug das Burn-out-Risiko 55\%.

Gesundheitliche Probleme und Krankschreibungen korrelierten signifikant mit dem Burn-out-Risiko. Kleinere gesundheitliche Probleme hatten im vergangenen Jahr $57 \%$ der Befragten. $32 \%$ von ihnen waren burn-out-gefährdet, $25 \%$ nicht. Von denen, die im vergangenen Jahr nicht einmal kleinere gesundheitliche Probleme hatten, also körperlich gesund waren, bestand lediglich bei $11 \%$ ein Burn-out-Risiko, und mehr als die Hälfte waren nicht burn-out-gefährdet, also auch seelisch gesund. 26\% aller Befragten waren im vergangenen Jahr krankgeschrieben. Die Burn-out-Gefährdung betrug bei denen, die sich hatten krankschreiben lassen, 35\%, bei denen, die sich nicht hatten krankschreiben lassen, nur 19\%. Von den durchschnittlich Burn-out-Gefährdeten ließen sich $28 \%$ krankschreiben.

Die Ergebnisse der Untersuchung zeigen, dass der Anteil kleinerer gesundheitlicher Probleme bei Erziehern und Lehrern in einer leitenden Position und solchen mit Untergebenenstatus vollkommen identisch ist. Das lässt darauf schließen, dass der körperliche Zustand der beiden Gruppen derselbe ist. Bei den Krankschreibungen dagegen zeigte sich ein signifikanter Unterschied zugunsten der Führungskräfte: Nur jeder zehnte Erzieher oder Lehrer in einer leitenden Position hatte sich krankschreiben lassen, während von denjenigen mit Untergebenenstatus im selben Zeitraum jeder Dritte krankgeschrieben war. 


\section{Diskussion}

Die im Jahr 2004 durchgeführte landesweite Untersuchung bei Pädagogen hatte gezeigt, dass die mentalen Merkmale der Pädagogen im Vergleich zum Durchschnitt der erwachsenen Gesamtbevölkerung und auch im absoluten Sinne positiver waren sowie mit denen anderer gesellschaftlicher Gruppen mit demselben demografischen und kulturellen Status übereinstimmten oder besser waren als deren Merkmale.

Anhand der repräsentativen landesweiten Untersuchung von PAKSI \&SCHMIDT (2006) haben die Forscher bei den Pädagogen - auf Grund des Deutlichwerdens des für das fortschreitende Lebensalter üblichen Musters - eine Signifikanz hinsichtlich der Durchschnittswerte der Burn-out-Skala nachgewiesen.

Mit der zunehmenden Dauer der Ausübung des Berufs erhöht sich die Wahrscheinlichkeit dessen, dass ein immer größerer Anteil der Burn-out-Symptome auftritt (PETRÓCZI et al. 1999). Anhand der Untersuchung in Westungarn wurde festgestellt, dass sich das Burn-out-Risiko mit der zunehmenden Dauer der Ausübung des Berufs nicht nur nicht erhöht, sondern verringert. Das liegt möglicherweise daran, dass die burn-out-gefährdeten Pädagogen, die die Schwierigkeiten nicht bewältigen können, ihren Beruf eher aufgeben, wodurch der Anteil der Gefährdeten in der jüngeren Generation höher und in der älteren Generation niedriger ist. Die Altersverteilung der Befragten aus Westungarn ist sowohl in ihrer Gesamtheit als auch hinsichtlich der Geschlechter gleichmäßig. PETRÓCZI (2007) zufolge beträgt der Anteil der Männer in diesen Berufen in den ersten 10 Jahren noch $11 \%$, verringert sich danach in der südlichen Region der Großen Tiefebene Ungarns jedoch rapide auf unter 5\%, was auf fehlende Anerkennung zurückzuführen ist.

Die Untersuchung in Westungarn hat gezeigt, dass die Dauer der Ausübung des Berufs mit der Dauer der Tätigkeit am aktuellen Arbeitsplatz identisch war. Das bedeutet, dass die Männer nicht den Arbeitsplatz wechseln, sondern sich eher innerhalb der Hierarchie bewegen. Das belegt auch der Umstand, dass 59\% der männlichen Befragten eine leitende Position innehatten.

Bei den Motivationen zur Berufswahl machen die Liebe zu Kindern 40\%, die Liebe zum Beruf 23\%, die Anregung durch die Eltern, die Familientradition und das familiäre Vorbild 7\% aus (PETRÓCZI et al. 2001). Die Ergebnisse der Untersuchung in Westungarn waren positiver: 53\% der Befragten gaben an, ihr Berufswunsch in der Kindheit sei der des Pädagogen gewesen. Bei 35\% gab es ein familiäres Vorbild und bei $20 \%$ lag sowohl der Berufswunsch als auch ein familiäres Vorbild vor. Das gleichzeitige Vorliegen eines familiären Vorbildes und des Berufswunsches war bei den Männern häufiger. Zugleich hatte das Vorbild der Mutter den größten Einfluss auf die Berufswahl.

Die Bedeutung der erzieherischen Attitüde der Eltern hinsichtlich der persönlichen Orientierung wurde belegt: Auch bei den Befragten war die vorherrschende elterliche erzieherische Attitüde eine übermäßig beschützende, überfordernde und akzeptierende. Nur jeder zehnte Erzieher oder Lehrer berichtete über eine gelegentlich akzeptierende, zurückweisende oder vernachlässigende elterliche Attitüde - die nach Roes Ansicht zu einer sachlichen Orientierung bei der Berufswahl führt. Die in der Kind- 
heit auftretende Ausrichtung des Arbeitsinteresses weist in die Richtung des pädagogischen Berufs. Die Befragten kennzeichnet die Dominanz des direktiven (29\%) und des sozialen (26\%) Interesses. Bei Pädagogen in leitenden Positionen herrscht die direktive Komponente vor (40\%), während die soziale und die innovative Ausrichtung (mit jeweils 21\%) gleich stark vorhanden sind. Bei den Männern ist die Dominanz der direktiven und der innovativen Komponente sehr ausgeprägt, bei den Frauen die direktive und die soziale Komponente. Pädagogen mit einem sachlichen und systematischen Arbeitsinteresse sind stärker burn-out-gefährdet als diejenigen mit einem sozialen, direktiven oder innovativen Interesse. Das wirkt sich auf den Unterricht aus. Erfolgreiche Lehrer sind eher zu Innovationen im Unterricht bereit. Erfolglose Lehrer nehmen die Ereignisse und die Situationen nicht angemessen wahr (FÜZI 2007).

Über die Population der befragten westungarischen Pädagogen kann man sagen, dass sie die Möglichkeit haben, fachliche Hilfe in Anspruch zu nehmen und diese auch zu nutzen. Der Grund für die hohen Teilnehmerzahlen bei Kursen und fachlichen Fortbildungsveranstaltungen kann sowohl in der kontinuierlichen Erneuerung des Berufs des Pädagogen als auch in dem mit dem Pädagogenberuf einhergehenden Anspruch, den neuen Herausforderungen gewachsen zu sein, liegen, und in dieser Hinsicht ist die Lage in Westungarn deutlich besser als in anderen Teilen des Landes.

Die relativ hohe Zahl der Teilnehmer an der Supervision und an Fallbesprechungen, die bei der Untersuchung festgestellt wurde, spiegelt die heute erwünschte Haltung wider, die einzelnen Einheiten unserer Arbeit (Schüler, Schülergruppen) nicht für sich, sondern als Teil eines Systems zu betrachten und sich im Klaren darüber zu sein, dass sich das ganze System ändert, wenn in der Funktion eines seiner Teile eine Veränderung eintritt.

Im Hinblick auf Erfolg, Entwicklung und Bewältigung ist wichtig, dass 92\% der Befragten angaben, die Möglichkeit zur Teilnahme an fachlichen Fortbildungsmaßnahmen sei gegeben. Die Absicht und der Bedarf der Teilnahme an solchen Veranstaltungen bestehen bei $89 \%$. Hier ist eine deutlich höhere Motivation zu beobachten als in Bezug auf die Veranstaltungen mit Trainingcharakter, bei denen es um die Persönlichkeit geht. Das liegt wohl daran, dass die fachlichen Fortbildungen einen eindeutigen Bezug zum Unterricht haben, die Unterrichtskompetenz steigern und einen bevorzugten Bereich der Arbeit der pädagogischen Tätigkeit betreffen, wie Borbála Paksi in ihrer Abhandlung über das landesweite Forschungsprojekt über Pädagogen erklärt. Die Programme und Trainings, die die Kompetenzen im Bereich der Persönlichkeit weiterentwickeln, dienen der Erhöhung der erzieherischen Kompetenz, die kein bevorzugter Bereich der pädagogischen Tätigkeit ist. Außerdem konnte festgestellt werden, dass die Motivation zur Teilnahme an Fortbildungen bei Erziehern und Lehrern generell gering ist (PAKSI \& SCHMIDT 2006).

Die intrinsischen Elemente der Motivation wurden belegt. Fachliche Anerkennung verringert das Burn-out-Risiko, fehlt sie jedoch, so erhöht sich dieses Risiko signifikant. Zusätzlich zur fachlichen Anerkennung verringert die finanzielle Anerkennung das Burn-out-Risiko, wobei die fehlende finanzielle Anerkennung die Burn-out-Gefährdung nicht erhöht. Moralische und menschliche Anerkennung führen nicht zu 
einem niedrigeren Burn-out-Risiko, das Fehlen moralischer und menschlicher Anerkennung hingegen erhöht dieses in beträchtlichem Maße.

Durch das konkrete Forschungsprojekt wurde die Aussage der Fachliteratur, laut welcher die Burn-out-Gefährdung und das Burn-out messbare physische, intellektuelle, soziale, psychologische und emotionale Symptome verursacht (PETRÓCZI 2007), eindeutig bestätigt. Die Beurteilung des Ausmaßes und der Auswirkungen der einzelnen Symptomgruppen war nicht Gegenstand des Forschungsprojekts, es wurde jedoch nachgewiesen, dass gesundheitliche Probleme und Krankschreibungen mit der Burnout-Gefährdung deutlich zunehmen. Ebenso wurde festgestellt, dass Pädagogen, die keine gesundheitlichen Probleme haben, auch seelisch gesund und nicht burn-out-gefährdet sind.

\section{Zusammenfassung}

Bezüglich des Zusammenhangs zwischen der Dauer der Ausübung des Berufs und dem Burn-out konnte die wichtige Feststellung gemacht werden, dass die Burn-out-Gefährdung mit zunehmender Dauer der Ausübung des Berufs nicht nur nicht zunimmt, sondern sich verringert. Der Grund dafür besteht meiner Ansicht nach darin, dass die burn-out-gefährdeten Pädagogen ihren Beruf eher aufgeben, sodass der Anteil der Gefährdeten in der jüngeren Generation höher und in der älteren niedriger ist.

Bei männlichen Pädagogen besteht eine beträchtliche Burn-out-Gefährdung. Die Untersuchung in Westungarn hat ergeben, dass bei vielen Befragten ein familiäres Vorbild vorliegt, das Frauen stärker beeinflusst. Die größte Bedeutung für die Berufswahl hat das Vorbild der Mutter. Ebenfalls von Bedeutung sind Tanten und Schwestern. Die Untersuchung hat gezeigt, dass die Möglichkeiten zur Vorbeugung gegen Burn-outs in der Region Westungarn vorhanden sind. Somit ist eine Änderung der Präventionsstrategie erforderlich, das heißt, den Pädagogen müssen keine weiteren Möglichkeiten angeboten werden, sondern sie müssen motiviert werden, die vorhandenen in Anspruch zu nehmen.

Die Ergebnisse des gegenständlichen Forschungsprojekts bestätigen den signifikanten Zusammenhang zwischen fachlicher Anerkennung, finanzieller Anerkennung sowie moralischer und menschlicher Anerkennung auf der einen und dem Burn-outRisiko auf der anderen Seite. Die finanzielle Anerkennung wirkt sich positiv auf die Burn-out-Gefährdung aus, ihr Fehlen erhöht sie jedoch nicht. Demgegenüber führt das Fehlen moralischer und menschlicher Anerkennung zu einer deutlich größeren Burn-out-Gefährdung, während die erhaltene moralische und menschliche Anerkennung keine Auswirkungen auf das Ausmaß des Burn-outs hat. Die gute Beziehung zu Kollegen und Mitarbeitern ist hinsichtlich des Burn-out-Risikos von Bedeutung, der wichtigste Faktor bezüglich der Gefährdung ist jedoch die Beziehung zum Vorgesetzten. Es wurde nachgewiesen, dass ein enger Zusammenhang zwischen körperlichen Symptomen und der Burn-out-Gefährdung besteht, weiterhin, dass körperlich gesunde Menschen auch seelisch gesund sind. 


\section{Referenzen}

BAgDY, E. (1997) 'Mi a szupervízió, az önismereti munka és pszichoterápia', Pszichoterápia $6,425-31$.

BODNÁR, G. (1998) Az emberi erőforrás fejlesztése az oktatásügyben (Budapest: Okker).

Coleman, M., L. Ganong \& K. LeON (2006) 'Divorce and Postdivorce Relationships' in A. Vangelisti \& D. Perlman, Hrsg., The Cambridge Handbook of Personal Relationships (Cambridge: Cambridge UP) 17-35.

CSIRSZKA, J. (1985) A személyiség munkatevékenységének pszichológiája (Budapest: Akadémiai).

DANCS, I. (1989) A pályamotivációt befolyásoló tényezők vizsgálata a szakoktatásban (Budapest: Akadémiai).

Edelwich, J. \& A. BRodsky (1980) Burnout: Stages of Disillusionment in the Helping Professions (New York: Human Services).

FrENKL, R. (2003) 'A sporttudomány és a mentálhigiéné' in T. TOMCSÁNYI, F. GREZSA \& I. JELENITS, Tanakodó: A mentálhigiéné elmélete, a mentálhigiénés képzés, mentálhigiéné az emberek szolgálatában (Budapest: Semmelweis Egyetem, Párbeszéd Alapítvány, Híd Alapítvány) 203-11.

FREUDENBERGER, H.J. (1974) 'Staff burn-out', Journal of Social Issues 30, 159-65.

FÜZI, B. (2007) 'Tanárok iskolai élményeinek szerepe a pedagógiai munka sikerességében', Alkalmazott pszichológia 9, 3-23.

GALlus, K. (1996) 'Segítő foglalkozásúak kiégési (burnout) szindrómájának megelőzése és gyógyítása csoportban', Pediáter 2, 132-42.

GeOSITS, B. \& B. KARDOS (1999) Kézikönyv pályaorientációs trénereknek (Vasvár: Reginnov).

HÉzSER, G. (2001) Miért? Rendszerelmélet és lelki gondozói gyakorlat: Pasztorál-pszichológiai tanulmányok (Budapest: Kálvin).

HorvÁth-SzABÓ, K. (2007) A házasság és a család belsö világa (Budapest: Semmelweis Egyetem).

KLEIN, S. (1990) Munkapszichológia a munkaszervezésben (Budapest: Tankönyvkiadó).

N. Kollár, K. \& É. SzABÓ (2004) Pszichológia pedagógusoknak (Budapest: Osiris).

ÓNODY, S. (2001) 'Kiégési tünetek (burnout szindróma) keletkezése és megoldási lehetőségei', Új Pedagógiai Szemle 6, 24-33.

PAKSI, B. \& A. SCHMIDT (2006) 'Pedagógusok mentálhigiénés állapota, különös tekintettel az iskolai értékátadást, egészségfejlesztést és problémakezelést befolyásoló dimenziókra’, $U_{j} j$ Pedagógiai Szemle 6, 12-26.

PÁLFFY, F. (2003) 'Szolgálat, önfeláldozás, hivatás? - A kiégés veszélyei ápolók körében’, Növér 6, 3-9.

PETRÓCZI, E. (2007) Kiégés - elkerülhetetlen? (Budapest: Eötvös József).

PETRÓCZI, E., M. FAZEKAS, Zs. TOMBÁCZ \& M. ZIMÁNYI (1999) 'A kiégés jelensége pedagógusoknál', Magyar Pszichológiai Szemle 3, 429-41.

PetrócZi, E., M. FAZEKAS, Zs. TOMBÁCZ \& M. ZIMÁNYI (2001) ‘A kiégés jelensége pedagógusoknál', Új Pedagógiai Szemle 6, 127-39.

RitoÓKNÉ, Á. M. (2000) A tanácsadás pszichológiája (Budapest: Nemzeti Tankönyvkiadó).

SAlAVECZ, Gy. (2008) 'Munkahelyi stressz és egészség' in M. KopP, Magyar lelkiállapot: Esélyerösités és életminőség a mai magyar társadalomban (Budapest: Semmelweis) 288-97.

SZILÁGYI, K. (1993) Tanácsadási elméletek (Gödöllő: GATE).

SZILÁGYI, K. (1997) Az egyéni tanácsadás: Módszertani kézikönyv a munkavállalási-, munka-, pályatanácsadók számára (Gödöllö: GATE).

SzILÁGYI, K. (2000) Munka-pályatanácsadás, mint professzió (Budapest: Kollégium). 